\title{
Identification by phage display of single-domain antibody fragments specific for the ODD domain in hypoxia-inducible factor 1alpha
}

Arjan J Groot ${ }^{1}$, Peter Verheesen ${ }^{2}$, Elike J Westerlaken ${ }^{1}$, Eelke H Gort ${ }^{1}$, Petra van der Groep ${ }^{1}$, Niels Bovenschen ${ }^{1}$, Elsken van der Wall ${ }^{3}$, Paul J van Diest ${ }^{1}$ and Avi Shvarts ${ }^{1}$

${ }^{1}$ Department of Pathology, University Medical Center Utrecht, Utrecht, The Netherlands; ${ }^{2}$ Department of Molecular and Cellular Biology, University of Utrecht, Utrecht, The Netherlands and ${ }^{3}$ Department of Internal Medicine, University Medical Center Utrecht, Utrecht, The Netherlands

\begin{abstract}
Hypoxia triggers the transcription of genes responsible for cell survival via the key player transcription factor hypoxia-inducible factor 1alpha (HIF-1 $\alpha$ ). Overexpression of this protein has been implicated in cardiovascular disorders, carcinogenesis and cancer progression. For functional and diagnostic studies on the HIF-1 $\alpha$ protein, we have identified single-domain antibody fragments directed against this protein by using a llama-derived nonimmune phage display library. This library displays the variable domains of the heavy-chain antibody subclass, found in these animals. Phage display selection with six recombinant HIF-1 $\alpha$ proteins yielded five different antibody fragments. By epitope-mapping, we show that all five antibody fragments bind within the functionally important oxygen-dependent degradation domain of the HIF-1 $\alpha$ protein. Two of these antibody fragments were engineered into bivalent antibodies that were able to detect human HIF-1 $\alpha$ by immunohistochemistry, Western blotting and immunoprecipitation, and mouse HIF-1 $\alpha$ by immunofluorescence and immunoprecipitation. These are the first single-domain antibody fragments that may be used in exploration of HIF-1 $\alpha$ as a possible therapeutic target through molecular applications.
\end{abstract}

Laboratory Investigation (2006) 86, 345-356. doi:10.1038/labinvest.3700395; published online 13 February 2006

Keywords: cancer; hetero-bivalent VHH; HIF-1 $\alpha$; hypoxia; phage display; single-domain antibody fragment; VHH

The half-life of hypoxia-inducible factor-1alpha protein $(\mathrm{HIF}-1 \alpha)$ is regulated by a sophisticated system of degradation, which involves binding to the von Hippel-Lindau tumor suppressor protein (pVHL), controlled by prolyl hydroxylases. During hypoxia, HIF- $1 \alpha$ forms a complex with HIF- $1 \beta$, resulting in an active HIF-1 transcription activation complex. Genes that contain a specific hypoxiaresponsive element (HRE) in their promoter sequences are activated during hypoxia in an HIF$1 \alpha$-dependent way. In order to secure cell survival, these hypoxia-responsive target genes are triggered to regulate metabolic adaptation to compensate for the reduced oxygen levels. ${ }^{1}$ This metabolic adaptation allows tumors, that reach a certain size and

Correspondence: Dr PJ van Diest, MD, PhD, Department of Pathology, University Medical Center Utrecht, PO Box 85500, 3508 GA Utrecht, The Netherlands.

E-mail: p.j.vandiest@azu.nl

Received 29 August 2005; revised 21 December 2005; accepted 23

December 2005; published online 13 February 2006 therefore outgrow their blood supply, to continue thriving with subsequent tumor progression and unfavorable outcome for the patient. ${ }^{2,3}$ High HIF-1 $\alpha$ expression plays a role in ischemic heart disease and different types of cancer, ${ }^{4,5}$ where HIF- $1 \alpha$ is considered to be a potential target for therapy. ${ }^{6-10} \mathrm{We}$ have shown the involvement of HIF-1 $\alpha$ in breast carcinogenesis $^{11}$ and in drug resistance. ${ }^{12}$ Furthermore, we have shown that high HIF-1 $\alpha$ expression, ${ }^{13}$ especially of the perinecrotic type ${ }^{14}$ correlates with bad prognosis. This latter finding has recently been confirmed by Dales et al ${ }^{15}$ in 745 breast cancer tissue samples. Studies on the role of HIF-1 $\alpha$ are, however, hampered by the small range of available antibodies. In this study, we have identified a set of llama heavy-chain antibody-derived, variable singledomain antibody fragments, also referred to as $\mathrm{VHH},{ }^{16-18}$ against both human and mouse HIF- $1 \alpha$. These $\mathrm{VHH}$ are the smallest naturally occurring intact soluble antigen-binding units with a molecular weight of about $\sim 15 \mathrm{kDa} .{ }^{16} \mathrm{VHH}$ are stable at high temperatures, ${ }^{19,20}$ can bind antigen in high salt 


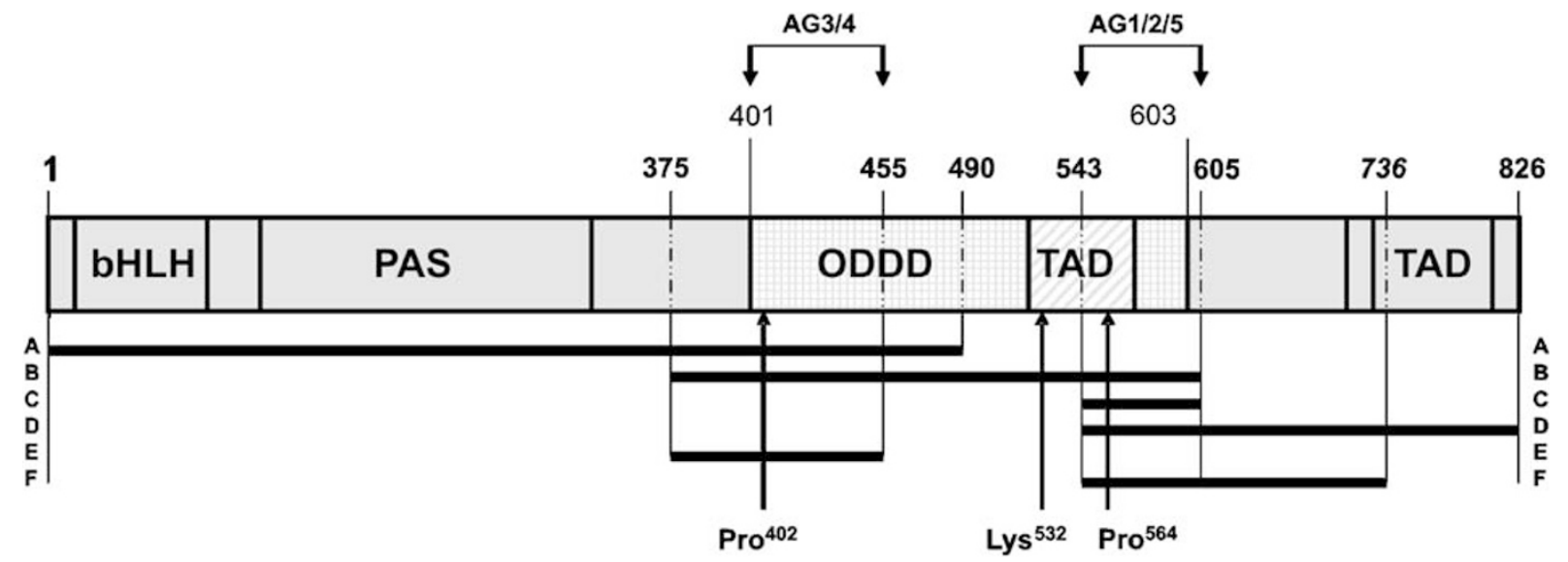

Figure 1 Schematic representation of the recombinant HIF-1 $\alpha$ fragments, used for selection of VHH. From top to bottom, A aa 1-490, B aa 375-605, C aa543-605, D aa543-826, E aa 375-455 and F aa 543-736, in correspondence to the amino-acid sequence of the human HIF-1 $\alpha$ protein. Pro ${ }^{402}$, Lys $^{532}$ and Pro ${ }^{584}$ in the ODDD (aa402-603) are the regulatory target residues. Approximate positioning of VHH AG1-5 recognition sites within the HIF-1 $\alpha$ protein are shown at the top of the figure.

concentrations, ${ }^{21,22}$ cripple intracellular viral replication, ${ }^{23}$ block enzymatic activity, ${ }^{24}$ are able to pass the blood-brain barrier, ${ }^{25}$ and can be used for various immunological applications like classical antibodies. We have selected a nonimmune $\mathrm{VHH}$ phage display library against six different recombinant HIF- $1 \alpha$ protein fragments. These fragments span across the human full-length HIF- $1 \alpha$ protein (Figure 1). One of the fragments partially represents a described alternative, transcriptionally active, splicing product of HIF- $1 \alpha$ : HIF- $1 \alpha$ isoform $2 .{ }^{26}$ Here, we report the identification of five novel monoclonal anti-HIF- $1 \alpha$ VHH. These VHH were mapped to epitopes within the oxygen-dependent degradation domain (ODDD), ${ }^{1,27}$ that contains both target sites of prolyl hydroxylation, ${ }^{28,29}$ essential for pVHL-dependent proteasomal degradation of the HIF-1 $\alpha$ protein (Figure 1). These anti-HIF-1 $\alpha$ VHH were engineered into higher affinity bivalent $\mathrm{VHH},{ }^{30,31}$ and validated for their use in several molecular techniques and histological studies.

\section{Materials and methods}

\section{Construction of Procaryotic Protein Production Vector pETAHIS}

For VHH selection strategies, a new procaryotic protein production vector was engineered. This vector allows the production of recombinant proteins with an N-terminal T7 tag and a C-terminal HIS6 tag. The pET-28a vector (Novagen, Madison, WI, USA) was modified for this purpose by removing the N-terminal HIS6 sequence. The rest of the original reading frame of pET-28a was unmodified and the optional C-terminal HIS6 tag was used for protein purification.

\section{Synthesis of the Recombinant HIF-1 $\alpha$ Fragments}

PCR with primers from Sigma-Genosys Ltd (Cambridge, UK), using pCEP4/HIF-1alpha ${ }^{32}$ plasmid as template resulted in the following products: fragment A coding for amino acids 1-490 of the human HIF-1 $\alpha$ protein was amplified using forward primer $5^{\prime}$-CGGGATCCATGGAGGGCGCCGGCGGCGCGAA-3' and reverse primer $5^{\prime}$-GGAGCGGCCGCGGTAAAA GAAAGTTCCAGTGAC-3'. All restriction enzymes and buffers were purchased from Roche (Basel, Switzerland). After digestion with BamHI and NotI, this fragment was ligated into the BamHI- and NotIdigested pET $\lambda$ HIS vector. Fragment $\mathrm{B}$ coding for amino acids 375-605 and fragment $E$ coding for amino acids $375-455$ of the human HIF- $1 \alpha$ protein were amplified with forward primer $5^{\prime}$-CGGGATCC CAGCTATTCACCAAAGTTGAATC- $3^{\prime}$ in combination with reverse primers 5'-GGAGCGGCCG CGTTAACTTGATCCAAAGCTCTG-3' and $5^{\prime}$-GGG AAGCTTGGTGGGTAATGGAGACATTGC- ${ }^{\prime}$, respectively. Fragment $\mathrm{C}$ encoding for amino acids 543-605 and fragment D for 543-826 of the human HIF- $1 \alpha$ protein were amplified with primer combinations forward 5'-CGGGATCCGACACAGAAG CAAAGAACCC- $3^{\prime}$ and with reverse primers $5^{\prime}$-GGG AAGCTTAGTCTGCTGGAATACTGTAAC- $3^{\prime}$ and $5^{\prime}$ GGGAAGCTTGTTAACTTGATCCAAAGCTCTG- ${ }^{\prime}$, respectively. The fragments were digested with BamHI and HindIII and cloned into a BamHI- and HindIII-digested pET $\lambda$ HIS vector. Fragment $F$ codes for amino acids $543-736$ of the HIF- $1 \alpha$ isoform $2^{30}$ and was amplified by PCR with the latter forward primer and reverse primer: 5'-GGGAAGCTTT CAAATAATTCCTACTGCTTGAAAAAG- $3^{\prime}$ The constructs were transformed into BL21(DE3)-RIL CodonPlus Competent cells (Stratagene, La Jolla, CA, USA) for protein expression. After induction with $1 \mathrm{mM}$ of isopropyl- $\beta$-D-thiogalactopyranoside 
(Sigma-Aldrich Co., St Louis, MO, USA), cells were lysed with Bacterial Protein Extraction Reagent (Pierce Co, Rockford, IL, USA). Recombinant fragments were purified with immobilized metal ion affinity chromatography (IMAC). Fragments that were insoluble were recovered using buffers with high urea contents. The produced recombinant HIF$1 \alpha$ fragments were dialyzed against PBS. Concentrations of the purified fragments were calculated from a BSA standard range using the $\mathrm{BCA}^{\mathrm{TM}}$ Protein Assay Kit (Pierce Co., Rockford, IL, USA). The fragments were checked by Western blot and Coomassie staining for the presence of tags and their purity. A construct with BamHI restrictions sites flanking full-length HIF-1 $\alpha$ was kindly provided by Professor G Simos ${ }^{33}$ and cloned into pET $\lambda$ HIS. This full-length recombinant HIF- $1 \alpha$ was also purified using IMAC and used in Western blot experiments. An expression construct coding for amino acids 401-603 (ODDD) of the HIF-1 $\alpha$ protein fused to the yellow fluorescence protein (YFP) was a kind gift of Dr T Brummelkamp and was used for immunoprecipitation-based epitope mapping.

\section{Selection of VHH}

The library used for VHH selections is a llamaderived nonimmune VHH library (Hermans et al, in preparation), which was kindly provided for this study by Unilever Research, Vlaardingen, the Netherlands. This phage display library was generated essentially as described before. ${ }^{34}$ Peripheral blood lymphocytes collected from the blood of eight nonimmunized llamas were used for mRNA extraction. cDNA was synthesized and VHH genes were PCR amplified introducing appropriate restriction sites for cloning. Phagemids carrying the $\mathrm{VHH}$ genes were then transformed into TG1 Escherichia coli and plated on selective plates. This resulting nonimmune library with a clonal diversity of $5 \times 10^{9}$ was used for phage preparations. VHH selections were performed according to an optimized selection protocol as described by Verheesen et al, (submitted for publication). ${ }^{35}$ This protocol utilizes different modes of antigen presentation to the phage antibody library that favors isolation of a diverse group of $\mathrm{VHH}$ against the antigen. HIF- $1 \alpha-$ specific VHH were selected in two successive rounds of selection utilizing T7-tagged recombinant-produced HIF-1 $\alpha$ fragments. A selection round with presentation of the antigen in solution by capturing with a T7-tag-directed monoclonal antibody was followed by a second selection round were direct panning of the recombinant fragments was used. The excess of noncaptured protein was removed by washing, and the coated antigens were incubated with the phage-VHH library. For capturing selections, $10 \%$ normal mouse serum (NMS) was added to the phage incubation to reduce binding of phage-VHH to the capturing agent. After removal of nonbinding phages by extensive washing, bound phages were eluted and rescued. At the end of selection procedures, enrichment for the six recombinant HIF-1 $\alpha$ fragments was observed. Ninty-six wells masterplates were picked from the corresponding second round phage outputs. ${ }^{36}$

\section{Screen for VHH}

For each masterplate, monoclonal VHH fragments were screened for their recombinant HIF-1 $\alpha$-binding ability in ELISA. To assess genetic diversity between positive clones, they were subjected to DNA fingerprint analysis ${ }^{36}$ and those with different digestion patterns were sequenced on an ABI3100 (Applied Biosystems Inc., Foster City, CA, USA).

\section{VHH Production}

VHH clones of interest were cultured, IPTG induced, and VHH were purified from the periplasm by using IMAC. ${ }^{36}$ Purified fractions were pooled and dialyzed to PBS. Concentrations of the purified VHH were calculated from a BSA standard range using the BCA ${ }^{\mathrm{TM}}$ Protein Assay Kit (Pierce Co, Rockford, IL, USA). The purified monoclonal VHH were stored at $-20^{\circ} \mathrm{C}$ for further use.

\section{Western Blot on HeLa Total Cell Lysate with Monovalent VHH}

Desferrioxamine (DFO) (Sigma) is an iron chelator that mimics the effect of hypoxia and stabilizes the HIF-1 $\alpha$ protein. ${ }^{37}$ All cell culture necessities were purchased from Gibco BRL (Paisley, UK). HeLa cells were grown to near confluency in $225 \mathrm{~cm}^{2}$ cell culture flasks and stimulated for $12 \mathrm{~h}$ with $0.1 \mathrm{mM}$ DFO or left untreated before lysis in $2 \mathrm{ml}$ of Laemmli loading dye. Both samples were sheared with a common insulin syringe and $50 \mu \mathrm{l}$ was loaded onto a $6 \%$ SDS-PAGE gel for separation of the proteins. Proteins were transferred to a PVDF membrane (Millipore Co., Bedford, MA, USA) and blots were blocked with 5\% MARVEL (dried skimmed milk, Premier International Foods, Coolock, UK) in PBS/ $0.025 \%$ Tween-20 (MPBST) at room temperature for $1 \mathrm{~h}$ and incubated overnight with $1 \mu \mathrm{g} / \mathrm{ml} \mathrm{VHH}$ in $15 \mathrm{ml}$ of $0.1 \%$ MPBS at $4{ }^{\circ} \mathrm{C}$. Next, the membranes were washed in PBS and incubated with a HRPconjugated mouse anti-6xHIS monoclonal antibody (BD Biosciences, San Diego, CA, USA) in $0.1 \%$ MPBS. ECL (Amersham Biosciences, Buckinghamshire, UK) was used for visualization as described by the manufacturer.

\section{VHH AG1 and AG2 Solid-Phase Competition Assay}

NUNC MAXISORP (NUNC, Roskilde, Denmark) plates were absorbed $\mathrm{o} / \mathrm{n}$ at $4^{\circ} \mathrm{C}$ with $\mathrm{VHH}$ AG1 $(0.48 \mu \mathrm{M})$ in $100 \mu \mathrm{l}$ per well in PBS. Wells were 
blocked with $200 \mu \mathrm{l} 1 \%$ BSA in PBS/0.1\% Tween-20 (BSAT) for $1 \mathrm{~h}$ at $25^{\circ} \mathrm{C}$ at $300 \mathrm{rpm}$ in a TERMOstar incubator (BMG LABTECH, Offenburg, Germany). All following incubation steps were performed under these conditions in this incubator. A nonrelated VHH (VHH-R2), which was kindly provided by Dr E Dolk, ${ }^{38}$ was used as a negative control in this experiment. Recombinant HIF- $1 \alpha$ fragment C $(4 \mu \mathrm{M})$ was incubated with immobilized VHH AG1 in the absence or presence of increasing concentrations of VHH AG1 or AG2 $(0-17 \mu \mathrm{M})$ in BSAT in a volume of $100 \mu \mathrm{l} 25^{\circ} \mathrm{C}$. After three rapid washes with PBST, bound recombinant HIF- $1 \alpha$ fragment $\mathrm{C}$ was detected by incubation with HRP-conjugated mouse anti-T7 tag antibody in a volume of $100 \mu \mathrm{l}$ (Novagen) (1:5000 in BSAT) for $20 \mathrm{~min}$, followed by staining using the ImmunoPure TMB Substrate kit (Pierce Co) according to fabricant instructions. Data were corrected for binding to empty microtiter wells, which was less than $5 \%$ relative to binding to wells containing immobilized VHH AG1. The signal intensities were measured using an ELISA reader (BioRad) at a wavelength of $450 \mathrm{~nm}$.

\section{Immunoprecipitation with Monovalent VHH}

HeLa cells grown to near confluence in $225 \mathrm{~cm}^{2}$ cell culture flasks were stimulated for $12 \mathrm{~h}$ with $0.1 \mathrm{mM}$ DFO, or left untreated. The cells were scraped and collected in $2 \mathrm{ml}$ immunoprecipitation buffer (IPB): $40 \mathrm{mM}$ Tris $\mathrm{pH}$ 8.0, 1\% Triton, 10\% Glycerol, $280 \mathrm{mM} \mathrm{NaCl}$ supplemented with a protease inhibitor cocktail (Roche). Collected cell lysates underwent three freeze-thaw cycles and were subsequently spinned down at $14000 \mathrm{rpm}$ for $10 \mathrm{~min}$ at $4^{\circ} \mathrm{C}$. In all, $200 \mu \mathrm{l}$ supernatant of both lysates was incubated with $1 \mu \mathrm{g}$ of VHH. Simultaneously, $1 \mu \mathrm{g}$ mouse anti-6xHIS monoclonal was bound (BD Biosciences, San Diego, CA, USA) to $7.5 \mu \mathrm{l}$ protein A/G plus-agarose beads (Santa Cruz Biotech, Santa Cruz, CA, USA) in $200 \mu \mathrm{l}$ IPB at $4^{\circ} \mathrm{C}$ head-over-head for $1 \mathrm{~h}$. Antibody-coated protein A/ $\mathrm{G}$ beads were blocked with $1 \%$ BSA in IPB at $4^{\circ} \mathrm{C}$ for 15 min and washed once with IPB buffer. Next, the preincubated VHH containing lysates were added to the beads and were incubated, head-over-head, overnight at $4^{\circ} \mathrm{C}$. Beads were washed 10 times with IPB and resolved in $50 \mu \mathrm{l}$ Laemmli loading dye. Samples were loaded onto a 6\% SDS-PAGE gel for separation of the proteins and analyzed with mouse anti-human HIF-1 $\alpha$ (clone 54, BD Transduction Laboratories, San Diego, CA, USA) and HRP-conjugated goat anti-mouse IgG + IgM (Biosource, Camarillo, CA, USA) by Western blot. All five VHH were tested to check whether binding sites were confined to the ODDD of the HIF- $1 \alpha$ protein. An expression construct coding for the ODDD of the HIF-1 $\alpha$ protein fused to YFP was transfected into HeLa cells with Lipofectamine ${ }^{\mathrm{TM}} 2000$ (Invitrogen, Carlsbad, CA, USA), according to the manufacturer's instructions. Cells were collected in IPB and immunoprecipitation protocol was continued as described above, replacing the 6xHIS for a mouse anti-Myc antibody (Invitrogen). For detection of the immunoprecipitated ODDD-YFP, a rabbit polyclonal anti-green fluorescent protein (anti-GFP, crossreacts with YFP, Santa Cruz Biotech, Santa Cruz, CA, USA) and HRP-conjugated goat anti-rabbit IgG $(\mathrm{H}+\mathrm{L})$ (BioRad, Hercules, CA) were used.

\section{Western Blot with Monovalent VHH against Recombinant HIF-1 $\alpha$ Proteins}

In total, $100 \mathrm{ng}$ of purified full-length recombinant HIF- $1 \alpha$ and recombinant HIF- $1 \alpha$ fragment B containing the ODDD domain of HIF- $1 \alpha$ were loaded onto a 6 and $12 \%$, respectively, SDS-PAGE gel by using a one-slot comb. Separated proteins were transferred to a PVDF membrane. Blots were blocked with $5 \%$ MPBST $/ 0.025 \%$ and incubated with $1.5 \mu \mathrm{g} / \mathrm{ml} \mathrm{VHH}$ in $0.1 \%$ MPBST at $4{ }^{\circ} \mathrm{C}$. Next, the membranes were washed in PBST and incubated with a mouse-anti- $C$ Myc monoclonal antibody (kindly provided by PW Hermans, Biotechnology Application Centre BV, Bussum, The Netherlands) and HRP-conjugated goat anti-mouse IgG + IgM. As a positive control, an HRPconjugated mouse anti-6xHIS monoclonal antibody was used. VHH-R2 was used as a negative control in this experiment.

\section{Determination of the VHH Binding Affinity to HIF-1 $\alpha$}

NUNC MAXISORP plates were absorbed o/n at $4^{\circ} \mathrm{C}$ with $100 \mu \mathrm{l}$ per well recombinant HIF- $1 \alpha$ fragment B at a final concentration of $5 \mu \mathrm{g} / \mathrm{ml}$ in PBS. Wells were blocked with $1 \%$ BSA in $200 \mu \mathrm{l}$ PBS/0.1\% Tween-20 (BSAT) for $1 \mathrm{~h}$ at $25^{\circ} \mathrm{C}$ at $300 \mathrm{rpm}$ in a TERMOstar incubator (BMG LABTECH). All the following incubation steps were performed under these conditions in this incubator. VHH were incubated at various concentrations $(0-18 \mu \mathrm{M})$ with immobilized HIF- $1 \alpha$ fragment $B$ in a volume of $100 \mu \mathrm{l}$ in BSAT for $1 \mathrm{~h}$ at $25^{\circ} \mathrm{C}$. After three rapid washes with PBST, bound VHH were detected by HRP-conjugated mouse anti-Myc antibody (Invitrogen) (1:5000 in BSAT) for $15 \mathrm{~min}$ at $25^{\circ} \mathrm{C}$. The signal intensities were measured using an ELISA reader (BioRad) at a wavelength of $450 \mathrm{~nm}$. The apparent affinities of the interaction between $\mathrm{VHH}$ and HIF- $1 \alpha$ were approximated from the determined concentration yielding half-maximum binding to HIF- $1 \alpha$ fragment B.

\section{Engineering of Mono- and Hetero-Bivalent VHH}

$\mathrm{X} 22$ is a VHH production vector for expression of cMyc and HIS6-tagged bivalent VHH. The encoded bivalent $\mathrm{VHH}$ are fusion proteins of two $\mathrm{VHH}$ in tandem. The vector is like the described $\mathrm{pKC}^{30}$ and 
shares the same plasmid backbone. In the X22 vector, the NCoI site is replaced by an SfiI site and the BstEII and NotI sites are interchanged. Also, the $\mathrm{X} 22$ has an additional c-Myc tag. A two-step cloning procedure was performed to create these bivalent VHH. The first step involves cloning of the C-terminal VHH in the PstI and BstEII sites of X22, followed by an SfiI and NotI cloning for introduction of the N-terminal VHH. To introduce a necessary PstI site, VHH were amplified by PCR with forward primers 5'-GGGCTGCAGATGGCC CAGGTAAAGCTGGA- $3^{\prime} \quad 5^{\prime}$-GGGCTGCAGATGGCC GATGTGCAGCTGGT-3', in combination with reverse primer $5^{\prime}$-CGCTTGCGGCCGCTGAGGA GACGGTGACCTG-3' $3^{\prime}$ by using the corresponding library phagemid vectors as template (clones AG1 and AG2). Reading frames were confirmed by sequencing. Monovalent VHH AG1 and AG2 were fused bivalent with their analog hinge and a 10 amino acid linker, AAAQVQLQMA, instead of the described $^{30}$ 'long hinge' linker. Purification was performed as described in VHH production. The result was a hetero-bivalent VHH AG-1N2C-MYC that was also recloned into the X22-VSV: a bivalent $\mathrm{VHH}$ production vector in which the $\mathrm{c}-M y c$ tag was replaced for a VSV-G tag (AG-1N2C-VSV).

\section{Recombinant HIF-1 $\alpha$ Fragment Competition Assay}

Protein A/G plus-agarose beads $(7 \mu \mathrm{l})$ were coated overnight with $20 \mu \mathrm{g}$ of purified hetero-bivalent VHH AG-1N2C-MYC in $200 \mu$ l IPB. Antibody-coated beads were blocked with $1 \%$ BSA-IPB at $4{ }^{\circ} \mathrm{C}$ for 15 min. DFO-stimulated HeLa lysates together with quantities of recombinant HIF-1 $\alpha$ protein fragments $\mathrm{C}$ and $\mathrm{E}$ were added to the coated beads, and the protocol was continued as described above in Immunoprecipitation with monovalent VHH. The presence of recombinant proteins in DFO-stimulated HeLa lysates was confirmed by Western blotting with the HRP-conjugated mouse anti-6xHIS monoclonal after separation of the total lysates on a $12 \%$ SDS-PAGE gel.

\section{Immunoprecipitation with Bivalent VHH AG-1N2C-VSV}

Purified hetero-bivalent VHH AG-1N2C-VSV (7 $\mu \mathrm{g})$ was incubated at $4{ }^{\circ} \mathrm{C}$ head-over-head overnight with $7 \mu \mathrm{l}$ protein A/G plus-agarose beads in $200 \mu \mathrm{l}$ IPB. Antibody-coated beads were blocked with $1 \%$ BSAIPB at $4^{\circ} \mathrm{C}$ for $15 \mathrm{~min}$ and washed once with IPB buffer before adding lysates of HeLa cells or NIH 3T3 cells and protocol was continued as described above in Immunoprecipitation with monovalent VHH. For detection of the mouse HIF-1 $\alpha$ protein, we used the rabbit anti-mouse HIF-1 $\alpha$ polyclonal (Novus Biologicals Inc., Littleton, CO, USA) and goat antirabbit IgG $(\mathrm{H}+\mathrm{L})$ HRP-conjugated (BioRad).

\section{Immunohistochemistry}

Paraffin-embedded material from renal cell cancer was obtained from the archives of the Department of Pathology, UMC, Utrecht. Immunohistochemistry was performed on 4- $\mu \mathrm{m}$ thick sections. All sections were dewaxed and rehydrated. For the detection of HIF-1 $\alpha$ with the hetero-bivalent VHH AG-1N2CVSV, antigen retrieval was performed in sodium citrate buffer $\left(\mathrm{pH} \mathrm{6.0)}\right.$ at $96^{\circ} \mathrm{C}$ for $20 \mathrm{~min}$. After a period of cooling down, the sections were incubated with the primary antibody (AG-1N2C-VSV; 40ng/ml) for $60 \mathrm{~min}$ at $20^{\circ} \mathrm{C}$. This was followed by the incubation with mouse-anti-VSV monoclonal (dilution $1 / 400$, Roche) for $60 \mathrm{~min}$ at $20^{\circ} \mathrm{C}$. The PowerVision histostaining kit (Poly-HRP-goat-anti-mouse/rabbit/ rat IgG, ready to use, ImmunoVision Technologies, Daly City, CA, USA) was used for detection. HIF- $1 \alpha$ staining was performed as described previously. ${ }^{11}$ Antigen retrieval was performed for $45 \mathrm{~min}$ at $96^{\circ} \mathrm{C}$ in target retrieval solution (DAKO, Glostrup, Denmark). The primary mouse antibody (anti-HIF-1 $\alpha$; 1/500 dilution, clone 54, BD Transduction Laboratories) was incubated for $30 \mathrm{~min}$ at $20^{\circ} \mathrm{C}$. The Catalyzed Signal Amplification System (DAKO) was used for detection. All sections were developed using diaminobenzidine, and subsequently counterstained with hematoxylin. Before the slides were mounted, all sections were dehydrated in alcohol and xylene.

\section{Immunofluorescence Microscopy}

NIH 3T3 mouse fibroblast cells were cultured on glass slides by using Dulbecco's modified Eagle's medium supplemented with $10 \%$ fetal calf serum, $100 \mathrm{U} / \mathrm{ml}$ penicillin-streptomycin and $100 \mathrm{U} / \mathrm{ml}$ L-Glutamine at $5 \% \mathrm{CO}_{2}$ and $37^{\circ} \mathrm{C}$. For the induction of HIF-1 $\alpha$, cells were stimulated with $0.1 \mathrm{mM} \mathrm{DFO} \mathrm{for}$ $12 \mathrm{~h}$. Slides were rinsed with PBS and cells were fixed with $3.7 \%$ formaldehyde-PBS for $10 \mathrm{~min}$. Cells were permeabilized with $0.1 \%$ Triton X-100 for $10 \mathrm{~min}$ and incubated with $50 \mathrm{mM}$ glycine-PBS for $10 \mathrm{~min}$ and blocked with $1 \%$ BSA-PBS for $30 \mathrm{~min}$. Subsequently, the cells were incubated with different concentrations $(2-100 \mathrm{ng} / \mu \mathrm{l})$ of hetero-bivalent $\mathrm{VHH}$ AG-1N2C-MYC at RT for $1 \mathrm{~h}$, followed by incubation with $0.44 \mathrm{ng} / \mathrm{ml}$ mouse-anti-c- $M y c$ monoclonal and $1 \mu \mathrm{g} / \mu \mathrm{l}$ DAPI in $1 \%$ BSA-PBS at RT for $1 \mathrm{~h}$. FITCconjugated polyclonal goat anti-mouse (DAKO, dilution $1 / 75)$ in $1 \%$ BSA-PBS was used for the detection. VectaShield (Vector Laboratories Inc., Burlinghame, CA, USA)-mounted microscope slides were assessed with fluorescence microscopy (LEICA DMR, Mannheim, Leica Geosystems, Germany).

\section{Results}

\section{Generation of Recombinant HIF-1 $\alpha$ Proteins}

Recombinant proteins produced from HIF- $1 \alpha$-specific constructs that span across the full-length HIF-1 $\alpha$ 
protein (Figure 1) resulted in six HIF-1 $\alpha$ fragments of expected molecular weight (data not shown). Fragments A, B, C, D and E were expressed with an N-terminal T7 and a C-terminal HIS6 tag. Fragment $F$ was expressed from the unmodified pET28a vector, which resulted in a recombinant protein with an N-terminal T7 and HIS6 tag. This fragment ends with an extra Isoleucine to distinguish from HIF- $1 \alpha$ isoform 2. Recombinant proteins B, C and E appeared in the soluble protein fraction; A, D and F appeared to be insoluble and were purified under denaturing conditions. The integrity and molecular weight of these fragments were determined with Coommasie blue staining and on Western blot using tag-specific antibodies. Homogeneity was >95\% (data not shown). These purified recombinant HIF$1 \alpha$ fragments were used for the selection of $\mathrm{VHH}$ from the phage display library.

\section{Selections and Screening for Anti-HIF-1 $\alpha$ VHH}

Significant enrichments in phage outputs were observed after two rounds of selection against different HIF- $1 \alpha$ fragments, except for fragment E. In total, 170 ELISA-positive clones for fragments A, $\mathrm{B}, \mathrm{C}, \mathrm{D}$ and $\mathrm{F}$ from selected $\mathrm{VHH}$ subpopulation were identified. Fingerprint analyses followed by sequence analyses of the $\mathrm{VHH}$ cDNA reading frames revealed that an identical VHH clone was selected against fragments $\mathrm{B}, \mathrm{D}$ and $\mathrm{F}$. These fragments contain an overlapping part of HIF- $1 \alpha$. This VHH was coded AG1. Surprisingly, from the selected phage subpopulation from the recombinant fragment C, a VHH with identical coding complementarity determining regions (CDR), but with different coding framework regions (FR), was identified as AG2 (Table 1). ${ }^{39}$ cDNA sequences between AG1 and AG2 differed even more (data not shown). Identification of AG2 validates the use of different fragments in our selection procedure. Had we used only fragment B for selection, we would never have identified VHH AG2 representing a completely different frame works as compared to AG1 (Table 1). We are the first to report selection of VHH with identical CDR and different FR from a nonimmune VHH phage display library.

\section{Competition of VHH AG1 and AG2 for Binding to HIF-1 $\alpha$}

We used a solid-phase competition assay to address the question whether or not VHH AG1 and AG2 can compete for binding to HIF-1 $\alpha$. As demonstrated in Figure 2, both monovalent VHH compete for binding to the recombinant fragment $\mathrm{C}$. These data imply that VHH AG1 and AG2 bind a similar region within HIF- $1 \alpha$.

\section{Screening of Anti-HIF-1 $\alpha$ VHH by Immunoprecipitation}

We used immunoprecipitation analysis to select for additional VHH that could recognize the native HIF$1 \alpha$ protein. This approach revealed three additional $\mathrm{VHH}$ that could recognize the native HIF-1 $\alpha$ protein: AG3, AG4 and AG5. VHH AG5 could also, like AG1, be identified from phage subpopulations corresponding to recombinant HIF- $1 \alpha$ fragments $B, D$ and $\mathrm{F}$ (Table 2). In addition, ELISA and Western blot crossreactivity experiments between these $\mathrm{VHH}$ and the recombinant HIF-1 $\alpha$ fragments showed that all five novel anti-HIF-1 $\alpha$ VHH could bind to their overlapping recombinant HIF- $1 \alpha$ fragments, as expected (Table 2). VHH AG1 and AG2 detected

Table 1 Selected anti-HIF-1 $\alpha$ VHH with FR1-4 and CDR1-3

\begin{tabular}{|c|c|c|c|c|}
\hline & FR1 & CDR1 & FR2 & CDR2 \\
\hline \multicolumn{5}{|l|}{ Name } \\
\hline \multirow[t]{2}{*}{ VHH } & & & & $52 \mathrm{~A}$ \\
\hline & 22 & 31 & 36 & 50 \\
\hline AG1 & QVKLEESGGGLVQPGGSLRLSCAASGSIS & INAMG & WYRQAPGKQREFVA & AIT TSGSTRYANSAKG \\
\hline AG2 & DVQLVESGGGLVQPGGSLRLSCAASGSIS & INAMG & WYRQAPGKQREFVA & AIT TSGSTRYANSAKG \\
\hline AG3 & AVQ̃LVDSGGGLVH PGGS LRLSCAASGS IF & INTMG & WFRQAAPGKEREFVA & AISGSNGKTYYADSVLG \\
\hline AG4 & QVQ̃LVESGGGLVQPGGS LRLS CAASGS IF & INAMA & WYRQAAPGKQRELVA & RIN RDGSTYADSVKG \\
\hline \multirow[t]{2}{*}{ AG5 } & EVQLVESGGGLVHPGGSLRLSCAASES IA & ASALG & WYRQIPG GRELVA & GIS SSGHTRYVDSVKG \\
\hline & FR3 & CDR3 & & FR4 \\
\hline \multicolumn{5}{|l|}{ Name } \\
\hline \multirow[t]{2}{*}{ VHH } & $82 \mathrm{ABC}$ & 10 & OABCDEFGH & \\
\hline & 66 & 95 & & 103 \\
\hline AG1 & RFTISRDNAKNTVYLQMDSLIPEDTAVYYCMS & RAFDRTI & EPWR & WGQGTQVTVSS \\
\hline AG2 & RFTISRDNAKNTVYLQ̃MNSLRPEDTAVYYCMS & RAFDRTI & EPWR & WGQ̃GTQ̃VTVSS \\
\hline AG3 & RSTISRDNAKSTVYLQMNSLKPEDTAVYYCAA & SGPYGS & VWLTGRSYDY & WGQGTRVTVSS \\
\hline AG4 & RFTISRDNAKRSVYLQMNSLKPEDTAVYYCNA & RQI ISH? & EY & WGQGNQVTVSS \\
\hline AG5 & RFTISRDNTENTVYLQMNSLKPEDTAVYYCRY & I GRPN & NV & WGQGTQVTVSS \\
\hline
\end{tabular}

Amino-acid sequence alignment of framework 1-4 and complementarity detemining region 1-3 for the five anti-HIF-1 $\alpha$ VHH; sequence numbering according to Kabat et al. ${ }^{39}$ Shaded: different amino acids in framework 1 and 3 of VHH AG1 and AG2. 
DFO-induced HIF- $1 \alpha$ at $120 \mathrm{kDa}$ on Western blot (Figure 3a and data not shown).

All identified VHH could immunoprecipitate the human native HIF- $1 \alpha$ and could detect recombinant HIF- $1 \alpha$ fragment $B$ as well as recombinant fulllength HIF-1 $\alpha$, using Western blot analysis (Figure $3 \mathrm{~b}$ and $\mathrm{c}$ ). The ODDD-YFP fusion protein was used to show that all described VHH bind specifically to an epitope in the ODDD of HIF- $1 \alpha$. All of the VHH were able to immunoprecipitate this fusion protein from HeLa cells, indicating that each one of those VHH recognizes epitopes located in this domain (Figure 4). Well-defined nonrelated VHH were used as a negative control in all of these experiments $(\mathrm{VHH}-\mathrm{R} 2){ }^{38}$

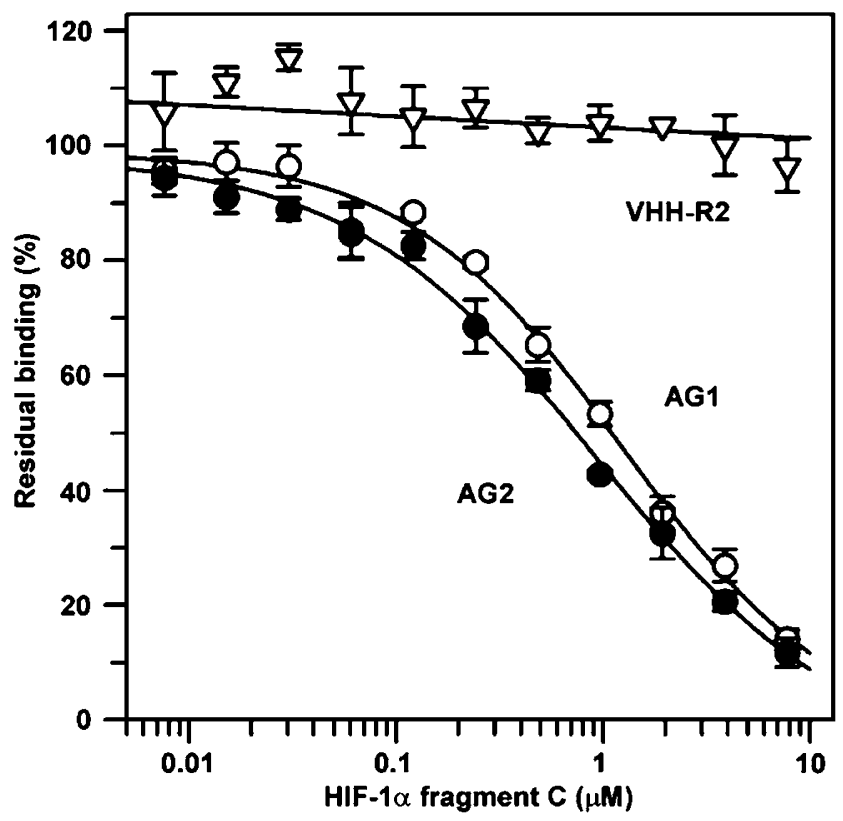

Figure 2 VHH AG1 and AG2 compete for binding to HIF-1 $\alpha$. Recombinant HIF-1 $\alpha$ fragment $C$ was incubated with immobilized VHH AG1 in the absence or presence of increasing concentrations of VHH AG1 and AG2. Residual binding is expressed as percentage of binding in the absence of competitor and is corrected for nonspecific binding. VHH-R2 was used as a negative control. Data represent the mean \pm s.d. of three experiments.

\section{Engineering of Bivalent VHH}

An additional advantage of the used phage display technique is that the coding cDNA sequence of the selected VHH is known. This feature makes these $\mathrm{VHH}$ easy to clone for other applications. $\mathrm{VHH}$ antibodies AG1 and AG2 showed a stronger signal on Western blot than the other selected VHH (Figure $3 \mathrm{~b}$ and c) and had the highest affinity (Figure 5). For this reason, we selected those two VHH to be cloned as bivalent $\mathrm{VHH}$. VHH were cloned in tandem, either with two identical $\mathrm{VHH}$ as mono-bivalent $\mathrm{VHH}$ or as hetero-bivalent VHH with combinations of different VHH. The four combinations we cloned were: AG1-AG1, AG2-AG2, AG1-AG2 and AG2AG1. All bivalent VHH combinations were expressed in E. coli. Differences in expression levels of bivalent $\mathrm{VHH}$, when isolated from the periplasm, were considerable. The bivalent VHH combinations with an N-terminal AG2 produced very poor. A combination of AG1 produced fair, but a combination of an N-terminal AG1 and a C-terminal AG2 produced exceptionally well. Both of these monovalent $\mathrm{VHH}$ recognize an identical epitope in the HIF- $1 \alpha$ region spanning amino acids $543-605$, represented in fragment $\mathrm{C}$, and this combination resulted in a hetero-bivalent VHH called AG-1N2C-MYC.

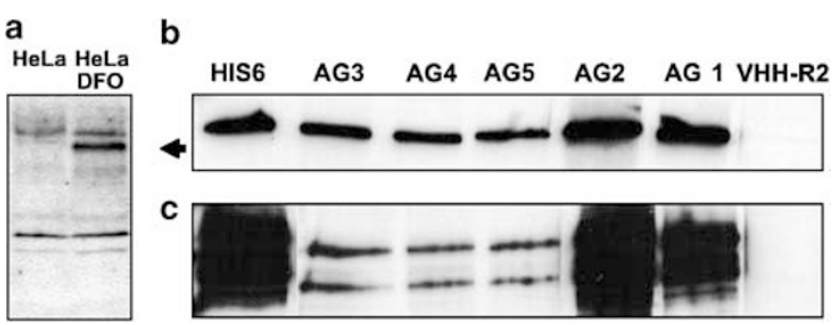

Figure 3 Detection of HIF-1 $\alpha$ by Western blot. (a) Western blot with monovalent VHH AG2 on non/DFO-stimulated HeLa cell lysates. An induced band representing the endogenous HIF- $1 \alpha$ protein is indicated by an arrow. Remaining bands are endogenous HeLa proteins recognized by the anti-6xHIS antibody. Western blot with the different anti-HIF-1 $\alpha$ monovalent VHH AG1-5, negative and positive control VHH-R2/HIS6 for (b) recombinant HIF- $1 \alpha$ fragment B (aa 375-605), and (c) recombinant full-length HIF-1 $\alpha$ (aa 1-826).

Table 2 VHH properties

VHH found in pool selected against recombinant fragmentDetection of recombinant HIF-1 $\alpha$ fragments A-F in ELISA/Western blot

$A$

Name

$\mathrm{VHH}$

AG1 BDF

AG2 C

AG3 A

AG4 A

AG5 BDF
A

$B$

C

$D$

$E$

F

$\begin{array}{llllll}- & + & + & + & - & + \\ - & + & + & + & - & + \\ + & + & - & - & + & - \\ + & + & - & - & + & - \\ - & + & + & + & - & +\end{array}$

VHH antibodies found in VHH subpopulations. Recombinant HIF- $1 \alpha$ fragment recognition of the five VHH in ELISA and Western blot experiments: cross-reactivity $+/-$ between VHH and recombinant HIF- $1 \alpha$ fragments. 


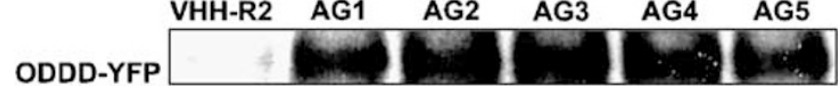

Figure 4 All selected VHH bind within the ODDD of the HIF-1 $\alpha$ protein. Immunoprecipitation from HeLa cells of an ODDD-YFP fusion protein by the different anti-HIF- $1 \alpha$ monovalent $\mathrm{VHH}$ AG1-5. As a negative control, VHH-R2 was used. All five VHH recognize specific epitopes within the ODDD of the HIF-1 $\alpha$ protein.

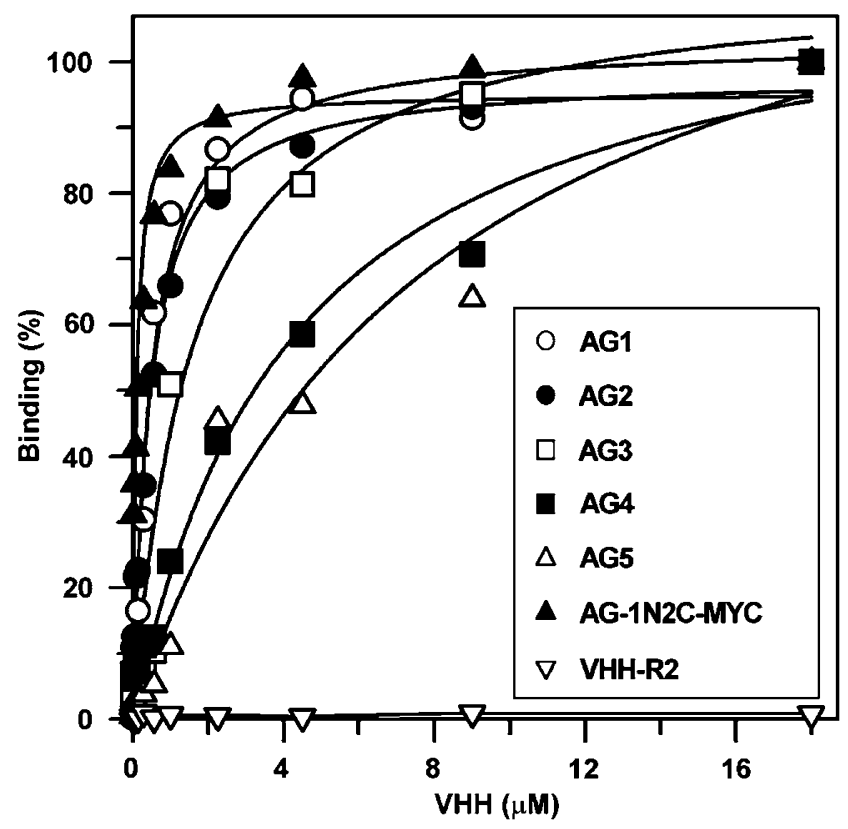

Figure 5 Apparent affinities for binding of VHH to HIF-1 $\alpha$. VHH were incubated with immobilized recombinant HIF-1 $\alpha$ fragment B. Binding is expressed as percentage of maximum binding for each VHH to recombinant HIF- $1 \alpha$ fragment B. The apparent affinity is approximated from the determined concentration yielding half-maximum binding to HIF- $1 \alpha$. VHH-R2 is used as a negative control. AG1 $\approx 0.48 \mu \mathrm{M}$, AG $2 \approx 0.45 \mu \mathrm{M}$, AG3 $\approx 1.82 \mu \mathrm{M}$, $\mathrm{AG} 4 \approx 4.26 \mu \mathrm{M}, \quad \mathrm{AG} 5 \approx 7.75 \mu \mathrm{M}$ and $\mathrm{AG}-1 \mathrm{~N} 2 \mathrm{C}-\mathrm{MYC} \approx 0.09 \mu \mathrm{M}$. Data represent the mean of duplicate experiments.

For this bivalent, VHH yields up to $20 \mathrm{mg} / \mathrm{l}$ were obtained. To show the specificity of hetero-bivalent VHH AG-1N2C-MYC for native HIF-1 $\alpha$ protein, a competition experiment was performed to verify once again specificity for native HIF- $1 \alpha$ after engineering of the bivalent VHH. DFO-stimulated HeLa lysates, with native HIF-1 $\alpha$, were incubated with two different recombinant fragments in an immunoprecipitation experiment. The fragments used were fragment E (aa375-455) and fragment C (aa543-605) without homology to and nonoverlapping with fragment E. Fragment $\mathrm{C}$ could compete with the native HIF-1 $\alpha$ protein for binding to the bivalent $\mathrm{VHH}$, as expected, because monovalent VHH used for cloning of the bivalent VHH preferentially bind to this fragment as has been shown in previous experiments (Table 2). This experiment indicates the specific binding of native $\mathrm{HIF}-1 \alpha$ protein with hetero-bivalent VHH AG-1N2C-MYC (Figure 6).

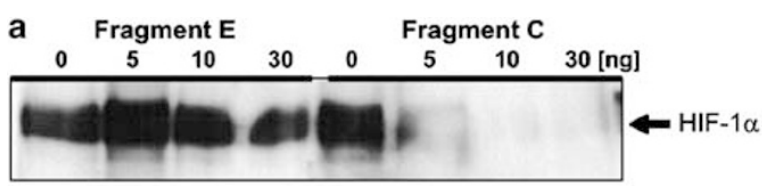

b

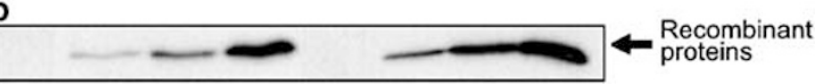

Figure 6 Bivalent VHH AG-1N2C-MYC is specific for native HIF$1 \alpha$. Recombinant HIF- $1 \alpha$ fragment $\mathrm{C}$ competes with native HIF- $1 \alpha$ for binding with hetero-bivalent VHH AG-1N2C-MYC in immunoprecipitation of HIF- $1 \alpha$ protein from DFO-stimulated HeLa cell lysates, indicating specific binding to the native HIF- $1 \alpha$ protein. Recombinant HIF- $1 \alpha$ fragment E, nonspecific for bivalent AG1N2C-MYC VHH did not compete at all with the native HIF-1 $\alpha$ protein. ng: range/amount of the recombinant HIF- $1 \alpha$ protein added. (a) Western blot for HIF-1 $\alpha$ detection. (b) Western blot for recombinant proteins $\mathrm{E}$ and $\mathrm{C}$ to illustrate how much of the recombinant protein was added to the HeLa cell lysates. Combined panels (a and b) show that the more fragment $\mathrm{C}$ is added, the less native HIF- $1 \alpha$ protein can be immunoprecipitated, as addition of fragment $\mathrm{E}$ to the lysate has no effect on the immunoprecipitation.

\section{Determination of the VHH Binding Affinity to HIF-1a}

To study the binding capacity of the VHH to HIF- $1 \alpha$, the apparent affinity was determined by calculation of half-maximum binding concentrations of $\mathrm{VHH}$ (Figure 5). The observed apparent affinities were $\approx 0.48 \mu \mathrm{M}$ for AG1, $0.45 \mu \mathrm{M}$ for AG2, $1.82 \mu \mathrm{M}$ for AG3, $4.26 \mu \mathrm{M}$ for AG4 and $7.75 \mu \mathrm{M}$ for AG5. The hetero-bivalent VHH AG-1N2C-MYC, a fusion of AG1 and AG2, had an apparent affinity of $0.09 \mu \mathrm{M}$. Consistent with the Western blot analysis (Figure $3 \mathrm{~b}$ and c), the highest apparent affinities are observed for VHH AG1 and AG2. In addition, the bivalent VHH AG-1N2C-MYC shows a five-fold increase of apparent affinity for binding to HIF- $1 \alpha$ as compared with the corresponding monovalent $\mathrm{VHH}$.

\section{Optimization of Human and Mouse HIF-1 $\alpha$ Protein Detection with VSV-Tagged Bivalent VHH}

The bivalent VHH AG-1N2C-MYC produced well in our bacterial expression system and is more efficient in the interaction with HIF- $1 \alpha$ as compared to the monovalent VHH. For increase of detection variety and avoidance of background signals, we chose to replace the c-Myc tag of the bivalent VHH AG-1N2CMYC by a VSV-G tag, which resulted in a heterobivalent VHH called AG-1N2C-VSV. This antibody could immunoprecipitate native human as well as mouse HIF- $1 \alpha$ protein from DFO-treated cell lysates (Figure 7).

\section{Endogenous Human HIF-1 $\alpha$ Detection by Immunohistochemistry}

Paraffin-embedded tumour sections of renal cell cancers that express mutant VHL protein and consequently high HIF- $1 \alpha$ protein levels due to 


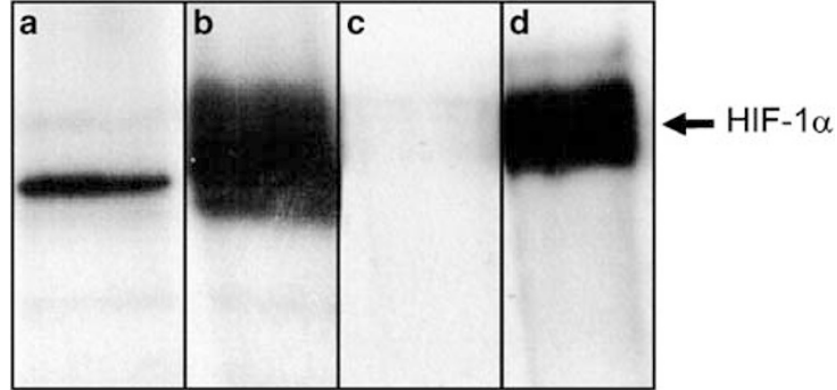

Figure 7 Hetero-bivalent VHH AG-1N2C-VSV is specific for the human and mouse HIF- $1 \alpha$ protein. Immunoprecipitation of the native HIF- $1 \alpha$ protein from human and mouse cell lysates with hetero-bivalent VHH AG-1N2C-VSV. In lane a, untreated NIH 3T3 lysate and lane b, DFO-stimulated NIH 3T3 lysates. In lane c, untreated HeLa lysates and lane (d), DFO-stimulated HeLa lysates. The band detected in lanes b (mouse) and d (human) corresponds to the induced endogenous HIF- $1 \alpha$ protein. impaired proteasomal degradation were used to test the usefulness of hetero-bivalent VHH AG-1N2CVSV in immunohistochemistry. This bivalent VHH could detect specific HIF-1 $\alpha$ nuclear staining in these tumors similar to a positive control antibody (clone 54, BD Transduction Laboratories) (Figure 8a and b).

\section{Endogenous Mouse HIF-1 $\alpha$ Detection with Anti-HIF-1 $\alpha$ VHH by Immunofluorescence Microscopy}

We investigated whether anti-HIF-1 $\alpha$ VHH was able to recognize endogenous mouse HIF- $1 \alpha$ in cultured cells with fluorescence microscopy. Upon DFO stimulation, stabilized HIF-1 $\alpha$ translocates to the nucleus and induces transcription of target genes. ${ }^{34}$ Indeed, we could visualize translocation of the
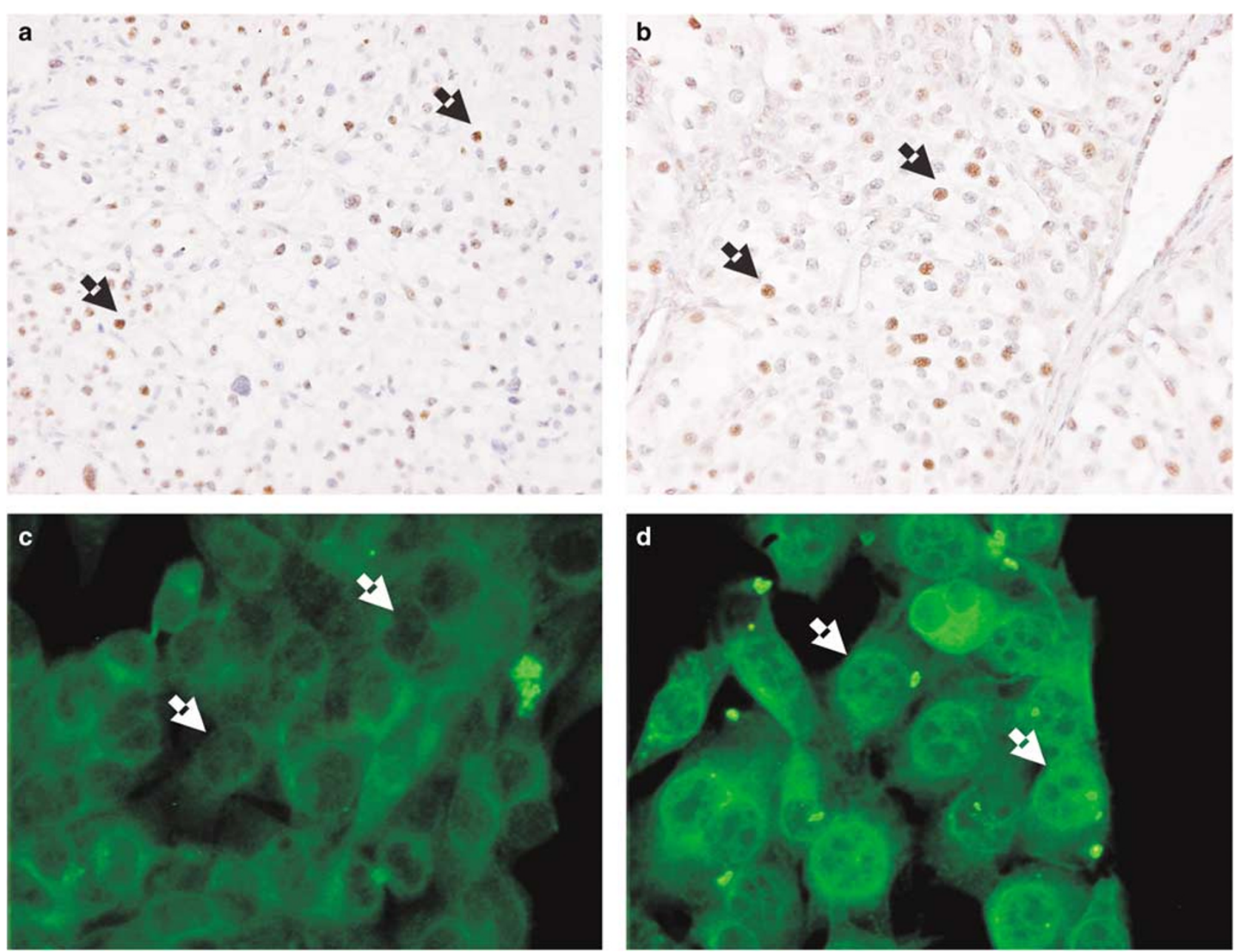

Figure 8 Hetero-bivalent VHH AG-1N2C-VSV detects specific nuclear signal in renal cell cancer specimens. Immunohistochemistry on paraffin-embedded slides with (a) commercial mouse monoclonal (BD Transduction Laboratories); (b) hetero-bivalent VHH AG-1N2CVSV. Both antibodies recognize identical nuclear HIF- $1 \alpha$ staining patterns. HIF-1 $\alpha$-negative nuclei stain blue and HIF- $1 \alpha$-overexpressing nuclei stain brown, as indicated by the arrowheads. Bivalent VHH AG-1N2C-MYC detects specific nuclear signal in NIH 3T3 cells after DFO stimulation, representing endogenous HIF-1 $\alpha$ protein. Immunofluorescence microscopy on cultured NIH 3T3 mouse fibroblasts. Cells were stained with $12.5 \mathrm{ng} / \mu$ l hetero-bivalent VHH AG-1N2C-MYC. (c) Untreated NIH 3T3 cells with negative nuclei indicated by the arrow heads. (d) DFO-stimulated NIH 3T3 cells with positively stained nuclei indicated by the arrow heads. 
mouse HIF- $1 \alpha$ protein into the nucleus in DFOstimulated mouse NIH 3T3 fibroblasts (Figure 8c and d). Both the monovalent VHH AG1 and AG2 could detect a similar nuclear signal (data not shown). In agreement with the observed higher affinity for HIF-1 $\alpha$ (Figure 5), hetero-bivalent VHH AG-1N2C-MYC could be used at a lower concentration as compared with monovalent VHH.

\section{Discussion}

Now that the human genome has been largely mapped, the next challenge will be to uncover the proteome. To this end, a wide range of specific antibodies to each protein is needed to detect the level, localization, splice variants and various post translational modifications that are crucial to protein function. As HIF-1 $\alpha$ is almost exclusively post-transcriptionally regulated, antibodies are of particular interest to study its function. In this manuscript, we describe the generation of llamaderived antibodies for diagnostics and molecular research on the HIF- $1 \alpha$ protein. Currently, available antibodies and their performance in certain immunological applications are mostly limited.

Selection of VHH against HIF- $1 \alpha$ by phage display, an important target in cardiovascular disease and human cancer, proved to be very successful. We identified five monovalent $\mathrm{VHH}$ able to recognize HIF- $1 \alpha$ within the ODDD (Figures 1 and 4). Some of these isolated $\mathrm{VHH}$ recognize epitopes more $\mathrm{N}$ terminal on the HIF- $1 \alpha$ protein than the commercial mouse monoclonal (clone 54, BD Transduction Laboratories, San Diego, CA, USA) that was used. Two of the monovalent VHH (AG1 and AG2) harbor identical CDR topped on different FR (Table 1) and competed for binding to HIF-1 $\alpha$ (Figure 2). This strongly suggests that both $\mathrm{VHH}$ recognize the same region within the ODDD. Fusion of AG1 and AG2 into a hetero-bivalent VHH markedly increased the binding affinity for HIF-1 $\alpha$ (Figure 5 ). We proved specificity of the engineered hetero-bivalent $\mathrm{VHH}$ AG-1N2C-MYC for the native human HIF-1 $\alpha$ protein by competition with recombinant fragment $\mathrm{C}$ (aa543-605) (Figure 6). This bivalent antibody detected mouse endogenous HIF- $1 \alpha$ protein in $\mathrm{NIH}$ 3T3 fibroblasts after DFO stimulation (Figure 8c and d). Furthermore, engineered hetero-bivalent VHH AG-1N2C-VSV was useful in immunohistochemistry of human renal cell cancer (Figure $8 \mathrm{a}$ and b) and could immunoprecipitate native human and mouse HIF-1 $\alpha$ protein (Figure 7). This is one of the first engineered antibodies recognizing mouse HIF-1 $\alpha$ that may be used in multiple molecular applications in human as well as mouse HIF- $1 \alpha$ model studies.

The significant advantage over conventional antibody generation is that phage display allows setting defined parameters, during selection, for the desired type of antibody. Selection strategies with altered presentation of the used recombinant HIF- $1 \alpha$ antigen proteins in $\mathrm{VHH}$ selection rounds in combination with the used nonimmune library showed to be very effective for isolating HIF-1 $\alpha$-specific monovalent VHH against the HIF- $1 \alpha$ protein. Furthermore, it generates infinite sources of monoclonal antibody.

The newly identified anti-HIF-1 $\alpha$ VHH were crossvalidated with a widely employed and accepted anti-HIF- $1 \alpha$ mouse monoclonal. This latter monoclonal antibody was raised against amino acids $610-727$ of the human HIF-1 $\alpha$ protein (clone 54, BD Transduction Laboratories, San Diego, CA, USA). Therefore, this commercial antibody would not recognize all splice products or homologs of the HIF-1 $\alpha$ protein. ${ }^{27}$ We might have limited ourselves, in the identified number of HIF-1 $\alpha$ binding VHH, by using this antibody in our validation screen by immunoprecipitation. Further investigation of the selected pool of a $170 \mathrm{VHH}$ from the present study may lead to the identification of $\mathrm{VHH}$ that can recognize these homologs and different splice variants of the HIF- $1 \alpha$ protein.

We attempted to isolate a VHH that could discriminate a one amino-acid difference ( $\mathrm{Ile}^{736}$ ) between isoform $2^{26}$ and the full-length HIF- $1 \alpha$ protein. However, we did not succeed due to the fact that recombinant fragment $F$ (543-736), which represents isoform 2, also contained an epitope that appeared to be very immunogenic to the used phage display library. This epitope is located in the overlap of recombinant fragments $\mathrm{B}, \mathrm{C}, \mathrm{D}$ and $\mathrm{F}$ (Figure 1). This is underlined by the fact that most of the available antibodies recognize the same confined region within the HIF- $1 \alpha$ protein. However, the use of phage display may allow selecting $\mathrm{VHH}$ to less-immunogenic epitopes. This could in theory be established by preclearing the VHH phage display library with recombinant fragment D (aa543-826), or by blocking the immunogenic epitope in recombinant fragment $F$ (aa543-736), with identified anti-HIF-1 $\alpha$ VHH AG1, AG2 and AG5 during selections. ${ }^{40,41}$

In our selection procedures, we used six recombinant HIF- $1 \alpha$ fragments, taking into account important amino-acid residues within the HIF- $1 \alpha$ protein, especially the residues in the ODDD spanning amino-acid sequence aa 401-603 $3^{1,8,27}$ (Figure 1). Fragments A (aa 1-490) and E (aa 375-455) contain amino acid residue $\mathrm{Pro}^{402}$ and fragments $\mathrm{C}, \mathrm{D}$ and $\mathrm{F}$ amino-acid residue $\mathrm{Pro}^{564}$. Fragment B (aa 375-605) contains both of these, important for hydroxylation targeted residues, necessary in regulation of HIF- $1 \alpha$ degradation. ${ }^{28,29}$ This fragment also contains aminoacid residue $\mathrm{Lys}^{532}$, a target for acetylation, also important in the regulation of the stability of HIF- $1 \alpha$ protein. ${ }^{42}$ We show that all selected VHH bind within the ODDD (Figures 1 and Figure 4). Therefore, binding of these VHH to HIF-1 $\alpha$ may block crucial functions of protein and may contribute to the elucidation of the functions of the HIF-1 $\alpha$ protein. If indeed interference with the functions of the HIF- $1 \alpha$ occurs, it may be worthwhile to 
explore the possible therapeutic potential of the identified anti-HIF-1 $\alpha$ VHH. Currently, we are investigating the effect on HIF-1 $\alpha$ stability and transcriptional activity by expressing these $\mathrm{VHH}$ intracellularly as intrabodies..$^{23,43}$

\section{Acknowledgements}

We thank Dr Hans de Haard and Professor Theo Verrips at the University of Utrecht for their stimulating guidance and advice throughout this work and Dr Theo van Laar for critical reading of this manuscript. This work was supported by The Dutch Cancer Society, grantnumber: UU2003-2825, and in part by the 1st and 2nd AEGON International Scholarship in Oncology.

\section{References}

1 Semenza GL. Regulation of mammalian $\mathrm{O}_{2}$ homeostasis by hypoxia-inducible factor 1. Annu Rev Cell Dev Biol 1999;15:551-578.

2 Brahimi-Horn MC, Pouyssegur J. The hypoxia-inducible factor and tumor progression along the angiogenic pathway. Int Rev Cytol 2005;242:157-213.

3 Ryan HE, Poloni M, McNulty W, et al. Hypoxiainducible factor-1alpha is a positive factor in solid tumor growth. Cancer Res 2000;60:4010-4015.

4 Semenza GL. HIF-1: mediator of physiological and pathophysiological responses to hypoxia. J Appl Physiol 2000;88:1474-1480.

5 Semenza GL. HIF-1 and human disease: one highly involved factor. Genes Dev 2000;14:1983-1991.

6 Brahimi-Horn C, Pouyssegur J. When hypoxia signalling meets the ubiquitin-proteasomal pathway, new targets for cancer therapy. Crit Rev Oncol Hematol 2005;53:115-123.

7 Escuin D, Simons JW, Giannakakou P. Exploitation of the HIF axis for cancer therapy. Cancer Biol Ther 2004;3:608-611.

8 Powis G, Kirkpatrick L. Hypoxia inducible factor1alpha as a cancer drug target. Mol Cancer Ther 2004;3:647-654.

9 Welsh SJ, Powis G. Hypoxia inducible factor as a cancer drug target. Curr Cancer Drug Targets 2003;3:391-405.

10 Park JW, Chun YS, Kim MS. Hypoxia-inducible factor 1-related diseases and prospective therapeutic tools. J Pharmacol Sci 2004;94:221-232.

11 Bos R, Zhong H, Hanrahan CF, et al. Levels of hypoxiainducible factor-1 alpha during breast carcinogenesis. J Natl Cancer Inst 2001;93:309-314.

12 Greijer AE, de Jong MC, Scheffer GL, et al. Hypoxiainduced acidification causes mitoxantrone resistance not mediated by drug transporters in human breast cancer cells. Cell Oncol 2005;27:43-49.

13 Bos R, van der Groep P, Greijer AE, et al. Levels of hypoxia-inducible factor-1alpha independently predict prognosis in patients with lymph node negative breast carcinoma. Cancer 2003;97:1573-1581.

14 Vleugel MM, Greijer AE, Shvarts A, et al. Differential prognostic impact of hypoxia induced and diffuse HIF- 1alpha expression in invasive breast cancer. J Clin Pathol 2005;58:172-177.

15 Dales JP, Garcia S, Meunier-Carpentier S, et al. Overexpression of hypoxia-inducible factor HIF-1alpha predicts early relapse in breast cancer: retrospective study in a series of 745 patients. Int J Cancer 2005;116:734-739.

16 Hamers-Casterman C, Atarhouch T, Muyldermans S, et al. Naturally occurring antibodies devoid of light chains. Nature 1993;363:446-448.

17 Dumoulin M, Conrath K, Van Meirhaeghe A, et al. Single-domain antibody fragments with high conformational stability. Protein Sci 2002;11:500-515.

18 Muyldermans S. Single domain camel antibodies: current status. J Biotechnol 2001;74:277-302.

19 Cortez-Retamozo V, Backmann N, Senter PD, et al. Efficient cancer therapy with a nanobody-based conjugate. Cancer Res 2004;64:2853-2857.

20 van der Linden RH, Frenken LG, de Geus B, et al. Comparison of physical chemical properties of llama VHH antibody fragments and mouse monoclonal antibodies. Biochim Biophys Acta 1999;1431:37-46.

21 Perez JM, Renisio JG, Prompers JJ, et al. Thermal unfolding of a llama antibody fragment: a two-state reversible process. Biochemistry 2001;40:74-83.

22 Dolk E, van der Vaart M, Lutje Hulsik D, et al. Isolation of llama antibody fragments for prevention of dandruff by phage display in shampoo. Appl Environ Microbiol 2005;71:442-450.

23 Dekker S, Toussaint W, Panayotou G, et al. Intracellularly expressed single-domain antibody against p15 matrix protein prevents the production of porcine retroviruses. J Virol 2003;77:12132-12139.

24 Desmyter A, Spinelli S, Payan F, et al. Three camelid $\mathrm{VHH}$ domains in complex with porcine pancreatic alpha-amylase. Inhibition and versatility of binding topology. J Biol Chem 2002;277:23645-23650.

25 Muruganandam A, Tanha J, Narang S, et al. Selection of phage-displayed llama single-domain antibodies that transmigrate across human blood-brain barrier endothelium. FASEB J 2002;16:240-242.

26 Gothie E, Richard DE, Berra E, et al. Identification of alternative spliced variants of human hypoxia-inducible factor-1alpha. J Biol Chem 2000;275:6922-6927.

27 Lee JW, Bae SH, Jeong JW, et al. Hypoxia-inducible factor (HIF-1) alpha: its protein stability and biological functions. Exp Mol Med 2004;36:1-12.

28 Jaakkola P, Mole DR, Tian YM, et al. Targeting of HIFalpha to the von Hippel-Lindau ubiquitylation complex by $\mathrm{O}_{2}$-regulated prolyl hydroxylation. Science 2001;292:468-472.

29 Ivan M, Kondo K, Yang H, et al. HIFalpha targeted for VHL-mediated destruction by proline hydroxylation: implications for $\mathrm{O}_{2}$ sensing. Science 2001;292: 464-468.

30 Els Conrath K, Lauwereys M, Wyns L, et al. Camel single-domain antibodies as modular building units in bispecific and bivalent antibody constructs. J Biol Chem 2001;276:7346-7350.

31 Cortez-Retamozo V, Lauwereys M, Hassanzadeh Gh G, et al. Efficient tumor targeting by single-domain antibody fragments of camels. Int J Cancer 2002;98: 456-462.

32 Forsythe JA, Jiang $\mathrm{BH}$, Iyer NV, et al. Activation of vascular endothelial growth factor gene transcription by hypoxia-inducible factor 1. Mol Cell Biol 1996;16:4604-4613. 
33 Chachami G, Paraskeva E, Georgatsou E, et al. Bacterially produced human HIF-1alpha is competent for heterodimerization and specific DNA-binding. Biochem Biophys Res Commun 2005;331:464-470.

34 Frenken LG, van der Linden RH, Hermans PW, et al. Isolation of antigen specific llama $\mathrm{VHH}$ antibody fragments and their high level secretion by Saccharomyces cerevisiae. J Biotechnol 2000;78:11-21.

35 Huang Y, Verheesen P, Roussis A, et al. Protein studies in dysferlinopathy patients using llama-derived antibody fragments selected by phage display. Eur J Hum Genet 2005;13:721-730.

36 van Koningsbruggen $\mathrm{S}$, de Haard $\mathrm{H}$, de Kievit $\mathrm{P}$, et al. Llama-derived phage display antibodies in the dissection of the human disease oculopharyngeal muscular dystrophy. J Immunol Methods 2003;279:149-161.

37 Wang GL, Semenza GL. Desferrioxamine induces erythropoietin gene expression and hypoxia-inducible factor 1 DNA-binding activity: implications for models of hypoxia signal transduction. Blood 1993;82: 3610-3615.

38 Spinelli S, Frenken LG, Hermans P, et al. Camelid heavy-chain variable domains provide efficient combining sites to haptens. Biochemistry 2000;39: 1217-1222.

39 Kabat E, Wu TT, Perry H, et al. Sequences of Proteins of Immunological Interest. US Department of Health and Human Service: Bethesda, 1991.

40 Ditzel HJ, Binley JM, Moore JP, et al. Neutralizing recombinant human antibodies to a conformational V2- and CD4-binding site-sensitive epitope of HIV-1 gp120 isolated by using an epitope-masking procedure. J Immunol 1995;154:893-906.

41 Sanna PP, Williamson RA, De Logu A, et al. Directed selection of recombinant human monoclonal antibodies to herpes simplex virus glycoproteins from phage display libraries. Proc Natl Acad Sci USA 1995;92:6439-6443.

42 Jeong JW, Bae MK, Ahn MY, et al. Regulation and destabilization of HIF-1alpha by ARD1-mediated acetylation. Cell 2002;111:709-720.

43 Verheesen P, de Kluijver A, van Koningsbruggen S, et al. Prevention of oculopharyngeal muscular dystrophy-associated aggregation of nuclear poly(A)-binding protein with a single-domain intracellular antibody. Hum Mol Genet 2006;15:105-111. 